http://jmscr.igmpublication.org/home/

ISSN (e)-2347-176x ISSN (p) 2455-0450

crossref DOI: https://dx.doi.org/10.18535/jmscr/v7i7.133

Journal Of Medical Science And Clinical Research

IGM Publication

An Official Publication of IGM Publication

\title{
Prevalence of Endometriosis in Unexplained Subfertile Women Diagnosed during Diagnostic Hysterolaparoscopy: A One Year Retrospective Study
}

\author{
Authors \\ Dr M. Santhi ${ }^{1}$, Dr Sundar Narayanan ${ }^{2}$ \\ ${ }^{1}$ Fellowship Resident, Endogynaecology, Siva Hospital \\ ${ }^{2}$ Professor, Dept of Endogynaecology, Siva Hospital
}

\begin{abstract}
Background: Endometriosis is a common condition seen in subfertile women especially in unexplained subfertility and incidence appears to increase in the recent era. This study aims to find out the prevalence of endometriosis in unexplained subfertile women diagnosed during diagnostic hysterolaparoscopy.

Settings and Design: A Hospital based retrospective study over a period of One year

Materials and Methods: It is a retrospective study conducted in the Rathna Fertility centre, Swamiyar madam, Tamil nadu from July 2018 to July 2019 among unexplained subfertile women undergoing diagnostic laparoscopy. A total of 75 patients underwent diagnostic hysterolaparoscopy for unexplained subfertility and out of these 34 patients who had laparoscopic evidence of endometriosis were included in the study.

Results: The frequency of endometriosis among women with unexplained subfertility subjected to diagnostic hysterolaparoscopy was found to be $45.3 \%$.

Conclusion: Endometriosis amongst subfertile women is increasingly being detected due to greater use of laparoscopy in evaluation of subfertility. Laparoscopy remains the gold standard for diagnosing and staging endometriosis. In unexplained subfertility, endometriotic spots were found in about half of the group undergone diagnostic laparoscope.
\end{abstract}

\section{Introduction}

Endometriosis is a gynaecological enigma since it is difficult to diagnose \& treat. It is an inflammatory disorder in women of reproductive age group, which is characterized by the presence of endometrial glands and/or stroma in sites other than the uterine cavity. It is an estrogendependent disease which usually involves the peritoneum or ovaries.

The prevalence of endometriosis has been estimated to be around $10-15 \%$ in the reproductive age women. Women with endometriosis can be either asymptomatic or can present with dysmenorrhoea, chronic pelvic pain, dyspareunia, subfertility \& adnexal mass. It has been seen that around $25-50 \%$ of subfertile women may be affected by endometriosis \& 30-35\% patients with endometriosis may suffer from subfertility.

Factors leading to reduced fecundity in mild endometriosis are impaired uterotubal transport of sperm, ovulatory disturbances, subtle impairment of oocyte \& embryo quality, implantation defects, antiendometrial antibodies, Progesterone resistance, increased risk of recurrent miscarriage. In advanced stages of endometriosis, it is mainly 
the distorted tubo ovarian axis along with above factors which contributes to subfertility.

The true pathophysiology of endometriosis is poorly understood. There are several theories explaining its pathophysiology which includes transplantation theory, induction theory, coelomic metaplasia theory, invagination theory, ovarian cyst theory \& vascular / Lymphatic spread.

Endometriosis is asymptomatic in about $1 / 5^{\text {th }}$ of the patients. The symptoms of endometriosis include severe dysmenorrhoea, deep dyspareunia, chronic pelvic pain, Dyschezia etc.

The role of ultrasound in the clinical diagnosis of endometriosis is of limited value as it lacks resolution for visualizing superficial peritoneal/ Ovarian imp lants (Endometriotic Spots) \& adhesions. Hence the Gold standard for diagnosis of endometriosis is laparoscopy, as it provides a visual proof of the minute Endometriotic lesions $\&$ helps in staging of the disease. In this study, we aim to find out the prevalence of endometriosis among unexplained Subfertile women without ultrasound evidence of endometriosis, subjected to diagnostic hysterolaparoscopy.

\section{Materials \& Methods}

This is a retrospective study conducted in Rathna Fertility centre, Swamiyarmadam, Tamil Nadu, for the period from July 2018 to July 2019. This study aimed to determine the prevalence of endometriosis in subfertile women without ultrasound evidence of endometriosis undergoing diagnostic hysterolaparoscopy for unexplained subfertility.

\section{Inclusion Criteria}

All patients of unexplained subfertility subjected to diagnostic hysterolaparoscopy who were diagnosed to have endometriosis were included in the study.

\section{Exclusive Criteria}

Subfertile women with ultrasound evidence of endometriosis or with other pathologies were excluded.
All patients included in the study were analysed with respect to the following characteristics.

\section{Clinical Characteristics}

Points noted were age \& duration of subfertility.

\section{Laparoscopic Findings}

Endometriotic lesions (Fig 1) which were noted varied from red, red-pink \& clear lesions/ White, yellow-brown \& peritoneal defects to black \& blue lesions. The size, depth $\&$ location of these lesions were noted to grade the severity of endometriosis. Endometriosis was classified by AFs in to 4 stages depending on the laparoscopic findings.

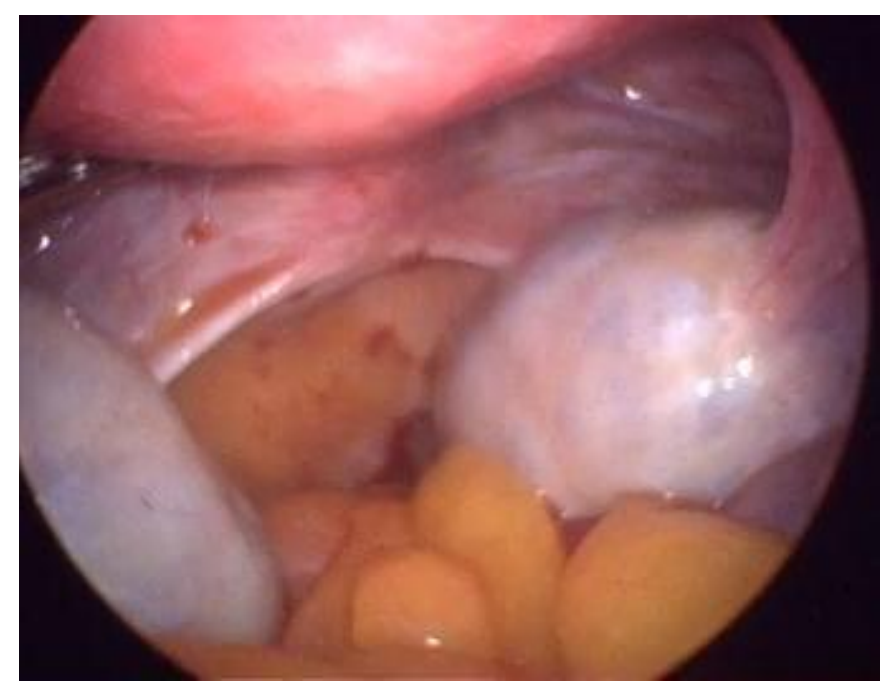

Fig 1

Stage I (Minimal) : 1-5

Stage II (Mild) : 6-15

Stage III (Moderate) : 16-40

Stage IV (Severe): > 40

\section{Unexplained Subfertility}

Unexplained subfertile groups are those with normal ovulation, patent tubes on HSG and husband's semen analysis showing normal semen parameters fulfilling recent WHO specification

\section{Results}

A total of 75 unexplained subfertile women without ultrasound evidence of endometriosis were subjected to diagnostic hysterolaparoscopy 
during the period from July 2018 to July 2019. Of these, $34(45.3 \%)$ patients had laparoscopic evidence of endometriosis. The mean age of patients was $30 \pm 5$ years (Range 20 to 40 years). Out of 34 patients who were diagnosed with Endometriotic spots on diagnostic hysterolaparoscopy, 19 patients were in the age group of 20-30years and 15 patients were in the age group of $31-40$ years. The duration of subfertility of the women ranged from 2 to 12 years. Out of 34 patients diagnosed with Endometriotic spots on diagnostic hysterolaparoscopy, there were 11 patients with 24 years duration of subfertility, 21 patients with 510 years duration of subfertility \& 2 patients with $>10$ years duration of subfertility. All patients diagnosed with Endometriotic spots were treated by Fulguration (Fig 2).

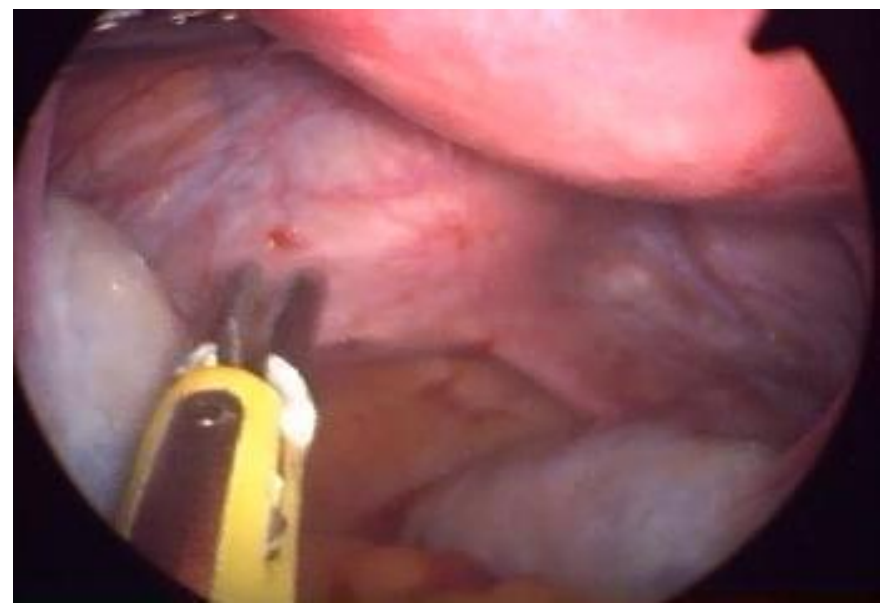

Fig:2

Table 2: Distribution of Endometriotic Spots Based on Age.

\begin{tabular}{|l|c|}
\hline Age ( Years) & No. Of Patients \\
\hline $20-30$ & 19 \\
\hline $31-40$ & 15 \\
\hline Total & 34 \\
\hline
\end{tabular}

Table 3: Distribution of Endometriotic Spots based on duration of Subfertility

\begin{tabular}{|l|c|}
\hline Duration of subfertility (Years) & No. Of Patients \\
\hline $2-4$ & 11 \\
\hline $5-10$ & 21 \\
\hline$>10$ & 2 \\
\hline Total & 34 \\
\hline
\end{tabular}

\section{Discussion}

Endometriosis affects $10-15 \%$ of women in the reproductive age group. It has been estimated that around $25-50 \%$ of subfertile women may be affected by endometriosis. The true incidence of endometriosis is difficult to establish since laparoscope is required for diagnosis of Endometriotic spots in patients who are asymptomatic . Our study demonstrates a higher incidence of Endometriotic spots (45.3\%) in unexplained subfertile women undergoing laparoscopy. The mean age of subfertile women in our study is $30 \pm 5$ years. Our study demonstrates a comparatively higher incidence of Endometriotic spots in the age group of 20-30 years (19 out of 34 patients) \& in women with a 5-10 years duration of subfertility (21 out of 34 patients).

Ultrasound lacks adequate resolution for visualizing Endometriotic implants or spots. Endometriotic spots can only be diagnosed by laparoscopy. All patients diagnosed with Endometriosis were treated by fulguration or cauterization of Endometriotic Spots by bipolar cautery. Thus this study highlights the higher prevalence of endometriosis in our population, particularly in asymptomatic subfertile females, with unexplained subfertility.

\section{Conclusion}

Endometriosis in subfertile females is not uncommon and is increasingly being detected because of greater use of diagnostic hysterolaparoscopy for evaluating subfertility. Ultrasound has limited value for diagnosing Endometriotic spots. Laparoscope remains the gold standard for diagnosing and staging endometriosis. More number of cases with Endometriotic spots than expected is diagnosed with laparoscope than by other modalities.

\section{References}

1. The infertility Manual by Dr. Kamini A Rao

2. Practice Committee of American Society for Reproductive medicine. Endometriosis 
and infertility: a committee opinion. Fertil Steril. 2012;98(3):591-98.

3. American Society for Reproductive Medicine. Revised American Society for Reproductive Medicine classification of endometriosis: 1996. Fertil Steril. 1997;67:817- 21 .

4. B Ranney. Endometriosis: IV Hereditary Tendency. ObstetGynaecol. 1971;37:734.

5. S Kennedy, A Berggquist, C Chapron, T D'Hooghe, G Dunselman, R Greb, et al. ESHRE guidelines for the diagnosis and treatment of endometriosis. Hum Reprod. 2005.

6. Novak's Text book of gynaecology - by Jonathan S Beret.

7. Te Linde's Operative Gynaecology - by Victoria. L.Handa / Linda VanLe 